Article

\title{
Selection of Optimal Path Control Algorithms for Probe Heads Used on Five-Axis Measuring Systems
}

\author{
Adam Gąska *D, Piotr Gąska, Maciej Gruza and Jerzy Sładek \\ Laboratory of Coordinate Metrology, Cracow University of Technology, 31-155 Kraków, Poland; \\ pjgaska@gmail.com (P.G.); gruzam@interia.pl (M.G.); sladek@mech.pk.edu.pl (J.S.) \\ * Correspondence: agaska@mech.pk.edu.pl; Tel.: +48-12-374-32-38
}

Received: 30 October 2018; Accepted: 22 November 2018; Published: 2 December 2018

check for updates

Featured Application: Results of this work may be directly applied by all users of probe heads with the possibility of continuous indexation for the reduction of measurement errors by the selection of optimal path control algorithms.

\begin{abstract}
The utilization of rotational movements of a probing system during points measurements contributes to the reduction of measurement duration and increases measurement repeatability. However, knowledge on such behavior and accuracy of probing systems is still unsatisfactory. Machines combined with articulating probing systems that have the ability of continuous indexation become redundant systems, which means that the same points can be measured using almost infinite mutual configurations of the machine and probe stylus orientations. Therefore, the proper selection of inspection path planning method becomes one of the main factors affecting the accuracy of the measurement. It is possible to assess the impact of this factor on the accuracy of the measurement by comparing the results of the measurements of gauge elements, which are done using different path controlling algorithms. After that, the best method for basic measuring tasks can be chosen in order to reduce measurement errors. Measurements of the multi-feature check gauge, using the default method for path planning and those chosen on the basis of described experiments, indicates that the improvement of accuracy may reach several microns. Results presented in this paper can be directly transferred to similar systems and measuring tasks, which are commonly met in industrial and scientific practice.
\end{abstract}

Keywords: five-axis system; CMM; dimensional measurements; inspection planning; accuracy

\section{Introduction}

\subsection{State of Art in Modern Coordinate Measurement Metrology}

Changes that can be observed in industry over the last years are commonly termed as a fourth industrial revolution. They are aimed at tightening the connection between individual components of the production process in order to reduce the manufacturing time and improve the quality of produced goods [1,2]. This trend is present in all industry branches connected with the production process, including quality control, especially coordinate metrology. Currently, the efforts of producers of measuring systems are focused mainly on the development of contactless techniques such as computed tomography (CT) or 3D scanners, often combined with industrial robots [3-5]. Their advantages are well known, most of all their high measurement speed and, in case of $\mathrm{CT}$, their possibility of part interior inspection. However, the accuracy of mentioned systems still cannot be compared to classic tactile coordinate measuring machines (CMMs), which for decades have been the key element of modern, automated quality control. 
Classic CMMs are still developed and enriched with solutions that improve their accuracy and efficiency. Utilization of the articulating probing systems that have the ability of continuous indexation (described in details in sub Section 1.2) fits in with this trend. A machine equipped with such a device becomes the five-axis system, capable of carrying out measurements with rotational movements of the probing system. In case of measurements of rotational features, it may cause a significant reduction of measurement duration and, for some measuring tasks, even accuracy improvement.

Figure 1 shows results of the experiment that involved reference ring measurements performed in two different modes on the same five-axis measuring system; once using only transitional moves of a CMM during probing, and once utilizing only the rotational movements of a articulating probing system. In both cases the applied measurement strategy was the same: The ring was measured in 32 points, distributed evenly across half of the reference ring's height, and, for both tested modes, measurements were performed 10 times. As can be seen, measurements performed with the rotation of the probing system reduced values of errors of form deviation measurements by nearly half, in relation to traditional three-axis measurements, and also contributed to the improvement in measurement repeatability.

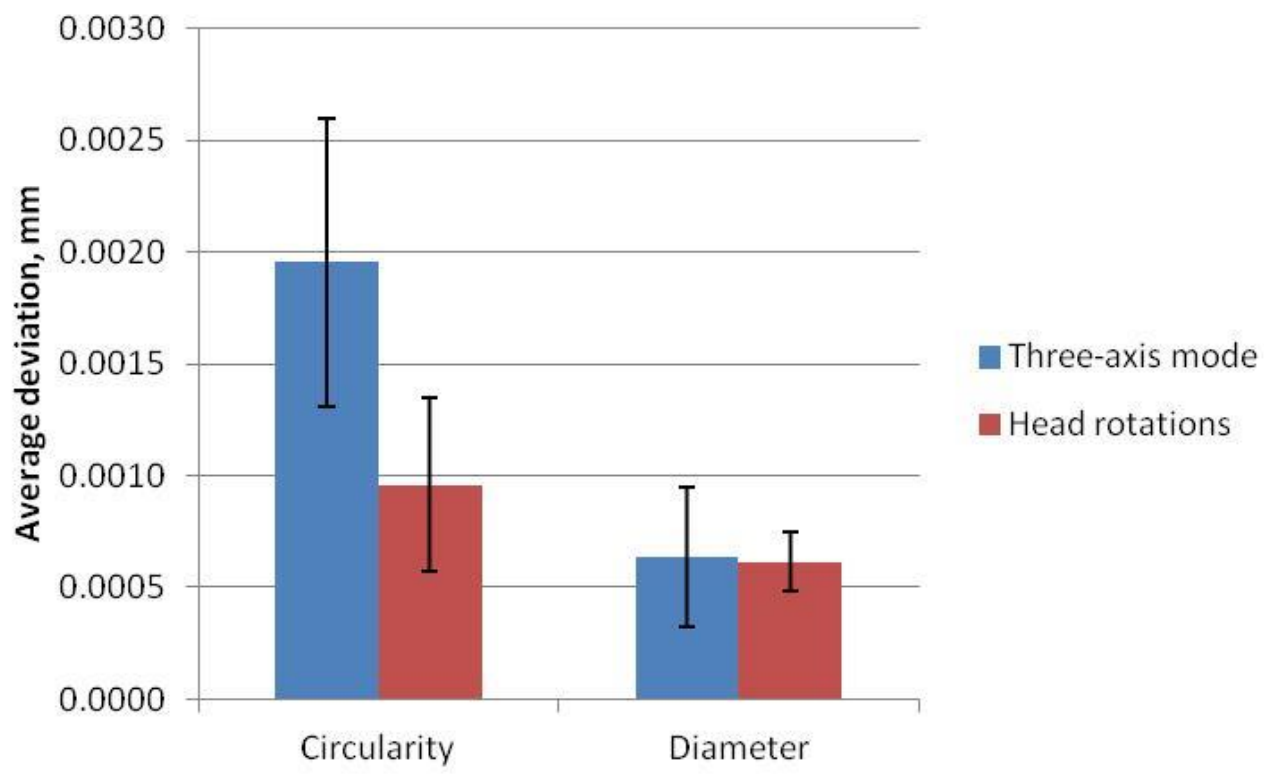

Figure 1. The results of the experiment showing the difference between measurements done using only the classic, three-axis coordinate measuring machine (CMM) and measurements done using measuring head movements. Error bars present the range of individual measurement results.

Additionally, it should be mentioned that the time needed to perform measurements with rotations of the probing system was two times shorter than in the case of the three-axis measurement. That results show that articulating probing systems are an interesting addendum to the possibilities of classic CMMs and they should be developed in the future. However, there are still many questions arising around this subject, with questions about articulating probing systems' accuracy and factors that may affect such probing systems' performance, being the most important ones. The software path control method, software correction, and algorithms used during measurement points determination are crucial for the proper operation of such measuring systems. This paper focuses on the first of mentioned factors, whereby we show the changes in measurement results depending on chosen path control method, and present how proper measurement strategy selection can contribute to the improvement of performance of the five-axis measuring system.

Considerations presented in this paper are topically related to the research on computer-aided inspection planning and the optimization of measurement strategy. There are a lot of papers focused on the optimization of the strategy of measurements performed on CMMs that use indexing probe 
heads ([6-8] to cite few of them), but there are no publications dealing with this topic for probe heads that have the possibility of continuous articulation.

\subsection{Five-Axis Coordinate Measuring System}

Articulating probing systems have been utilized in CMM measurements for many years. They allow the ability to change the orientation of the stylus using two rotations around mutually perpendicular axes. Two configurations are used in their construction. In the first approach, the axis running through the stylus coincides with the probing system's vertical axis of rotation (axial adjustment); whereas in the second solution, the axis running through the stylus is parallel to the vertical axis of rotation of the probing system, and offset from it at a specified distance (side adjustment). The first solution is regarded as more accurate; however, on the contrary to the second approach, it has a limited range for rotation about the horizontal axis of a probe. Another possible classification of articulating probing systems can be found in [9], dividing them depending on the qualification process. For some probing systems the experimental qualification is needed for all orientations used during measurement; however, in the second option qualification is done only in several positions and then interpolated for any orientation. Despite the used solution, articulating probing systems working with classic CMMs have a fixed orientation during coordinate measurements, which is kept by a locking mechanism, or by the servo control system. The stylus orientation can be changed before or after point probing, but actual measurements are done only using translational moves of machine elements. Additionally, it should be noted that usually the producers specify the step of the angular increment for possible rotations, for example $2.5^{\circ}, 7.5^{\circ}$, or $15^{\circ}$. The reason for the requirement of the proper operation of the articulating probing systems is the high repeatability of angular positioning. Otherwise, any change in orientation would entail the requalification of the probe head.

In five-axis measuring systems the articulating probing system becomes a crucial element of the whole device. Such a probe can remain fixed in a chosen orientation, but its rotary motions can also be utilized during measurements. In that case, the data set needed for point coordinate determination is expanded by information about the angular position of the probe head, which is given by angular encoders. The probe heads utilize axial adjustment, with two orthogonal axes of rotation. The range of possible rotations for the vertical axis of revolution, which will be called $\mathrm{B}$ axis in the rest of the paper, is $-180^{\circ}$ to $180^{\circ}$, and for the horizontal axis, which will be called A axis in the rest of the paper, the range from $-115^{\circ}$ to $115^{\circ}$. The probing system is oriented vertically (along the machine quill) when $A$ and $B$ angles are set to $0^{\circ}$. Both measuring and touch trigger probes can work as a part of the five-axis measuring system, and both kinds use the same kinematics. One of the main differences between articulated probe heads used in three-axis CMMs and those utilized in five-axis measurements is the continuous indexation of the head. As the articulated probes use rotary moves for measurements, it must be possible to set the probe orientation freely within the working range of the probe. This is realizable thanks to the qualification process designed especially for such probing systems. It is divided into two steps: Firstly, the geometrical calibration of the head (as it is named by producers of such devices) is performed, then the probe is qualified. Both procedures are similar and based on calibration sphere measurements. However, the first procedure is done with short styli, to minimize bending and gravitational effects and, in turn, to accurately assess the geometrical errors of the head mechanism, whereas the second procedure is done for stylus and stylus tip configuration, which are chosen for measurements. The steps comprised in the procedures includes: The determination of calibration of ball position in the three-axis mode for the probe directed vertically, with $\mathrm{B}$ angle set to $0^{\circ}$, and then the second time with $B$ angle set to $180^{\circ}$; the determination of probing parameters, such as probing speed and maximal acceleration (this step is done without standard measurements); and the determination of the interpolated probe map, based on sphere measurements using head rotational movements, performed for different angular orientations of the probe head and for different cross-sections of the standard. Both parts of the qualification process take a considerable amount of time (more than $10 \mathrm{~min}$ for the Renishaw PH20 probe head), but they do not have to be repeated, in the case of the probe, until 
a new probe configuration is needed, and in the case of the head geometrical calibration, until the probe head would be disassembled from the machine quill.

The problem of the articulating probing system's accuracy was studied mostly for systems with fixed indexation, retained during probing. Described aspects of this problem include angular positioning repeatability [10], general accuracy [11,12], and probing system modelling [13]. Fewer studies have been focused on a probing system with continuous indexation, mostly done in the Laboratory of Coordinate Metrology (LCM), among them research has been done about such probing system's hysteresis and the influence of stylus length [14] and the angular position of the probing system on measurement accuracy [15]. Conducted experiments revealed the existence of additional problems worth further examination; one of them is the issue of selecting the appropriate control algorithm for the measurement path for measurements utilizing the rotational movements of the probing system. This problem will be investigated in the following chapters of this paper.

\section{Path Control Algorithms of the Articulating Probing System}

The software responsible for path control and the determination of measured points coordinates plays a crucial role in the proper performance of the five-axis coordinate system. Most metrological programs allow the five-axis coordinate system to be used as a classic CMM equipped with a articulating probe, which retains a fixed orientation during point probing. Currently the most popular software, which allows the ability to fully use the rotational moves capabilities of a probing system, is Modus software by Renishaw. The programming environment is based on dimensional measuring interface specification (DMIS), but it is also possible to combine it with external applications in order to enrich the software capabilities. To control the probing system's performance, Modus uses an option called "tilt" and "advance", which defines the orientation of the probe during measurements. Tilt defines the rotation of the probe away from the plane, perpendicular to the measured surface normal vector [16]; and advance specifies the rotation around surface normal, while the negative value of this parameter means that the head is behind the tip [16]. As can be seen, the definitions are not exactly clear. However, the code created in the DMIS format uses, directly, the values of the A and $\mathrm{B}$ angles during measurement, or utilizes the Euler angles notation when the orientation is given in the part coordinate system (PCS). During the measuring path determination, Modus visualizes the location of the measured points, and the probe's stylus orientation corresponding to them, by drawing a leader line from each measured point to the probing system's central point [16]. Depending on the geometrical feature chosen for measurement, four different methods can be applied to manipulate the probe orientation: Guide point (GP), guide rail (GR), guided curve (GC), and fixed tilt and advance (FTAA). All mentioned methods are shown in Figure 2.

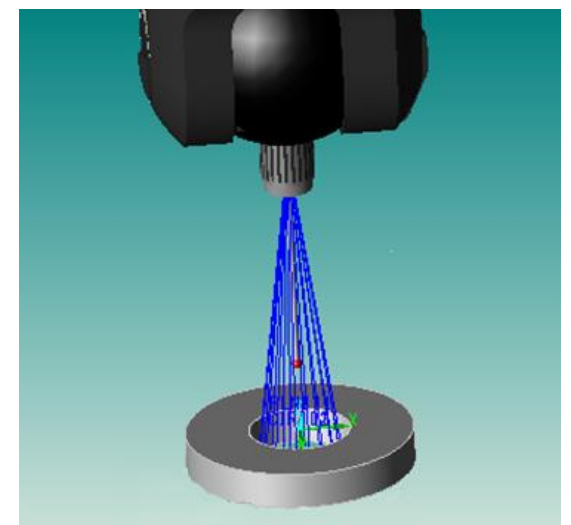

(a)

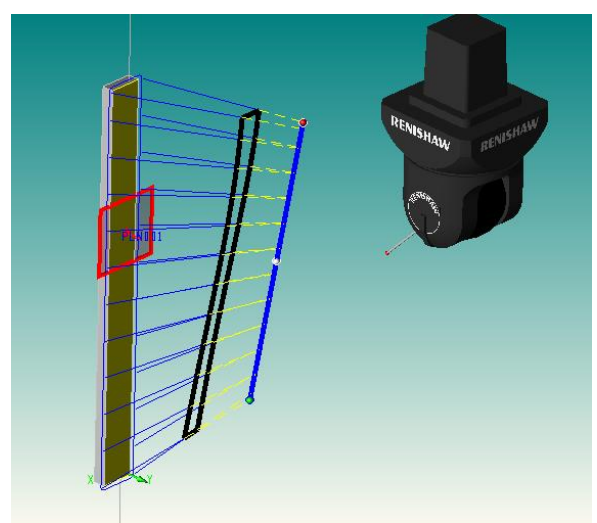

(b)

Figure 2. Cont. 


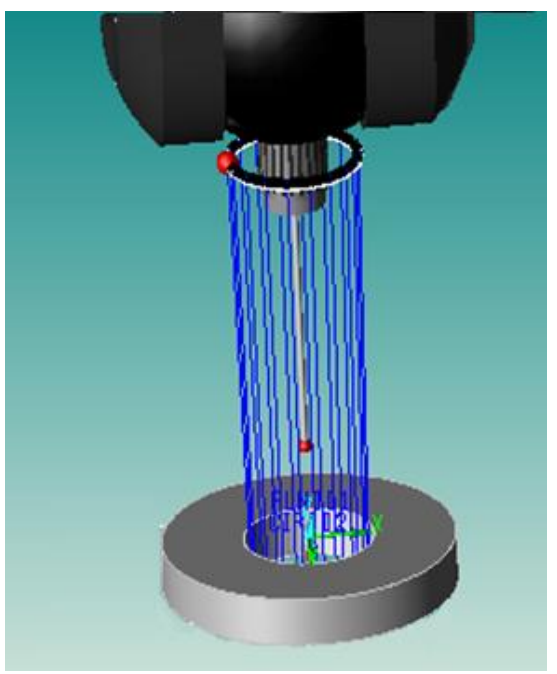

(c)

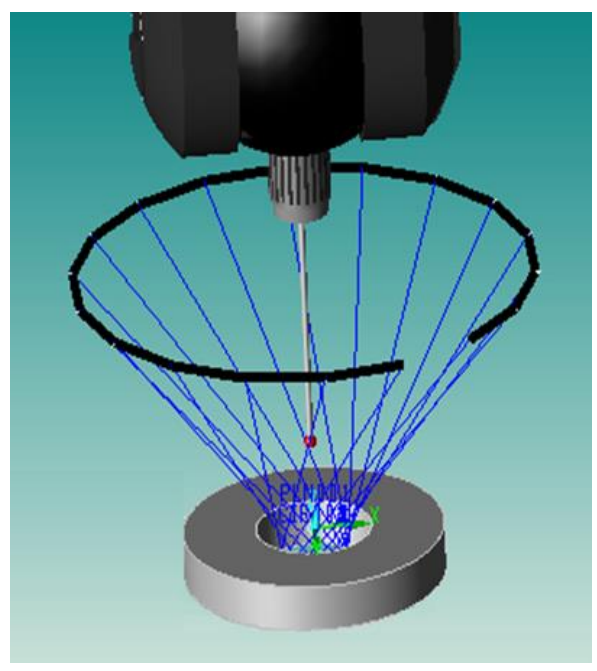

(d)

Figure 2. Path control algorithms used during five-axis measurements: (a) Guide point (GP); (b) guide rail (GR); (c) guided curve (GC); and (d) fixed tilt and advance (FTAA).

Guide point is the option used for minimizing machine motion during probing. The centroid, which determines the position of the center point of the probing system, is calculated from points included in the inspection path. It is offset along the normal direction to the plane in which the measured points are defined. The user can also specify the position to be taken by the head during measurements. The guide rail method can be used to define the path for the center point of the probing system during measurement. It consists of two segments between three reference points, of which the position can be defined by the user. By default, the position of the starting and ending points of the rail is calculated as offset, in normal direction to the plane, on which the measured points lie at the two extreme points of the inspection path. The offset has the same length as the utilized stylus. The guided curve is calculated, taking into account all points in the inspection path, by projecting them along the normal direction to the plane, on which the inspection path lies. Fixed tilt and advance can be used to ensure that each measured point would be inspected using the same tilt and advance values. In the case of the measurement of rotational features, it results with big changes in the probing system's center point position during measurements. Table 1 shows which method can be applied for the measurements of different geometrical elements.

Table 1. Path control algorithms that can be applied for measurement of specified features.

\begin{tabular}{|c|c|c|c|c|c|}
\hline Feature & $\begin{array}{c}\text { Guide Point } \\
\text { (GP) }\end{array}$ & $\begin{array}{l}\text { Guide Rail } \\
\text { (GR) }\end{array}$ & $\begin{array}{c}\text { Guided Curve } \\
\text { (GC) }\end{array}$ & $\begin{array}{c}\text { Fixed Tilt and } \\
\text { Advance (FTAA) }\end{array}$ & Comments \\
\hline Point & + & - & - & - & \\
\hline Line & - & + & + & - & \\
\hline Plane & + & + & - & - & \\
\hline Circle & + & - & + & + & \\
\hline Cylinder & + & - & + & + & \\
\hline Sphere & - & - & - & - & $\begin{array}{l}\text { "Tilt and advance" } \\
\text { option unavailable }\end{array}$ \\
\hline Surface & - & - & - & - & $\begin{array}{l}\text { "Tilt and advance" } \\
\text { option unavailable }\end{array}$ \\
\hline
\end{tabular}

\section{Experiment and Results}

All measurements described in this article were performed on a five-axis measuring system, which was part of the LCM's equipment. It consisted of a CMM with a moving bridge, Zeiss WMM 850S (ZEISS International, Oberkochen, Germany), and the articulating probing system, PH20 by Renishaw 
(Wotton-under-Edge, UK), working with the TP20 STD probe. The whole system was located in an air-conditioned room, with constant ambient conditions that were monitored. The temperature during experiments varied between 19.8 to $20.4{ }^{\circ} \mathrm{C}$. The experiments undertaken at the LCM consisted of two parts: The first part was conducted to assess the influence of the different methods used for path control on the measurement accuracy. It involved multiple measurements of standard objects; gauge block of $200 \mathrm{~mm}$ length and gauge ring of $28 \mathrm{~mm}$ diameter (Figure 3).

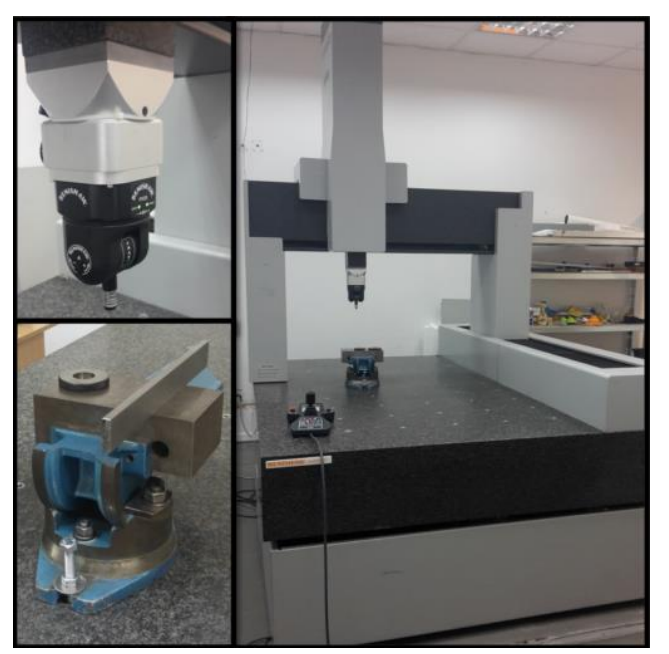

Figure 3. Photographs of the Zeiss WMM 850S (right side), the Renishaw PH20 measuring head (upper left), and the mounting of gauges during measurements (lower left).

The first step of the gauge block measurements was the determination of the local coordinate system, which was defined using three mutually perpendicular planes of gauge (the measurement of the planes that define the part coordinate system were performed using the standard three-axis mode, so only transitional movements of the machine were used during probing). After this step, the distance, angle between planes, parallelism, and flatness deviations were measured using different methods for inspection planning. Two methods were included in this part of the experiment, guide point and guide rail, as only these two were available for measurement of planes. Additionally, three different orientations of the guide point were utilized, marked as $0^{\circ}, 45^{\circ}$, and $90^{\circ}$ (denoted respectively as GP $0^{\circ}$, GP $45^{\circ}$, and GP $90^{\circ}$ on Figures 4 and 5) with names corresponding to values around which A angle used during probing oscillated. Measurements performed with all mentioned methods were repeated 10 times and if it was possible in all cases the measurements were done with the same distribution of measured points. The results of measurements are presented in Figure 4 which shows average deviations from nominal values (taken from calibration certificate of gauge block) and in Figure 5 which gives information about standard deviation for each measurement task.

The most noticeable differences between the results obtained for the different path planning methods during gauge block measurements can be seen for the measurements of the angle and parallelism deviation. The smallest difference was obtained for measurements of flatness. In most cases the lowest values of standard deviation can be observed for the path control algorithms that also gave the lowest values of deviations. 


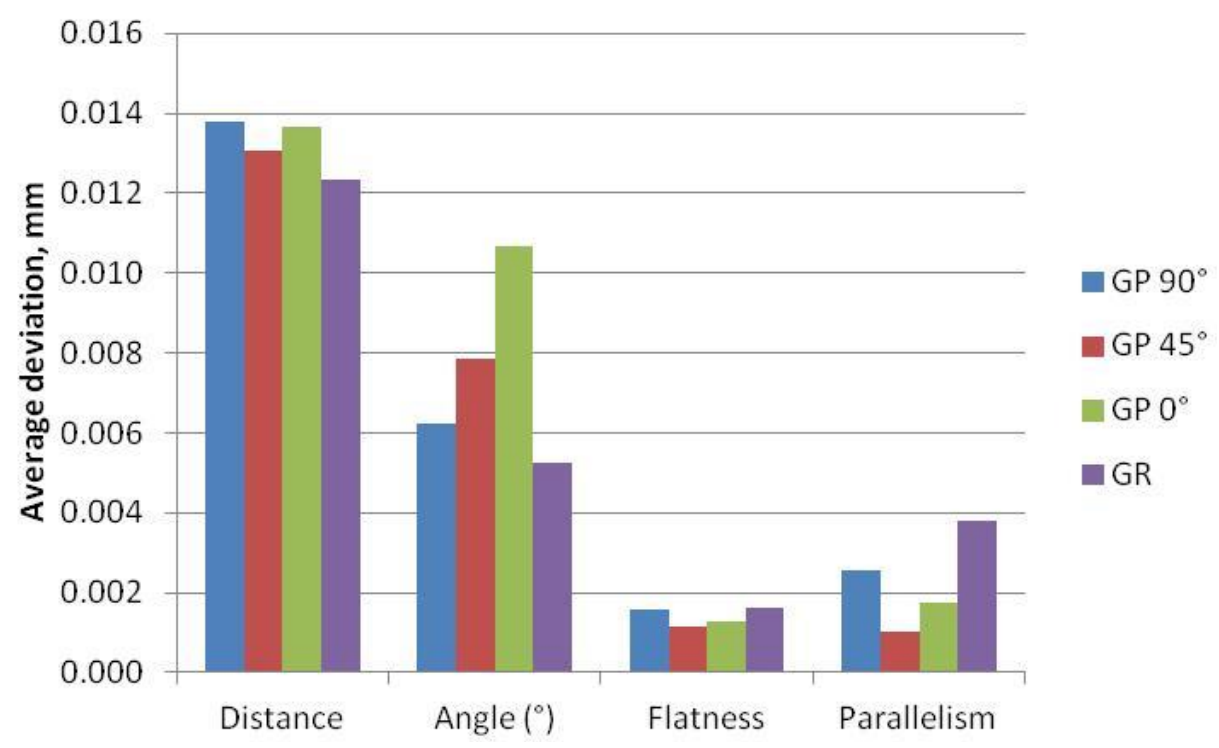

Figure 4. The average deviations for the four chosen tasks, measured on the gauge block using different path control algorithms.

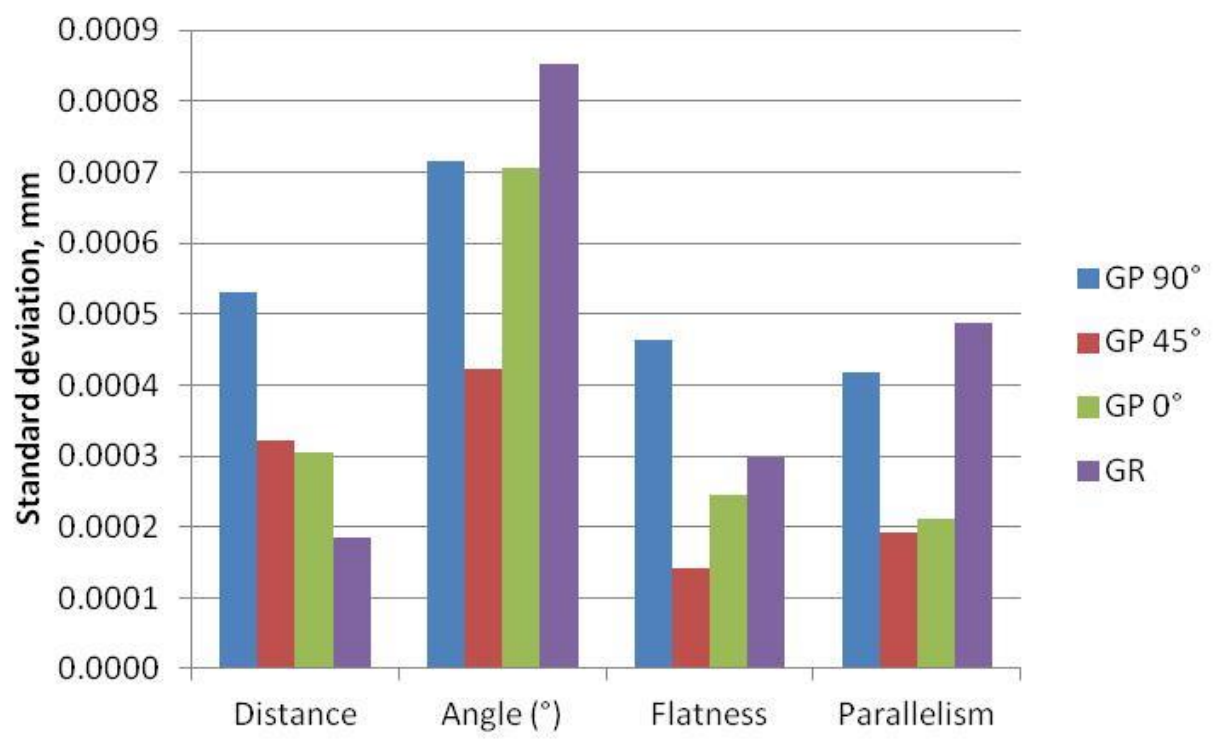

Figure 5. The standard deviations for the four chosen tasks, measured on the gauge block using different path control algorithms.

The next part of the experiment involved measurements of the ring gauge using all possible methods of inspection path planning. The local coordinate system was created in the center of the ring, with the ring's axis determining the spatial alignment of the coordinate system. Again, the measurement needed for the coordinate system definition was performed in the three-axis mode of the measuring system. The ring was measured across half of its height, in 16 evenly-distributed points. Three available methods were tested: Guide point, guide curve, and fixed tilt and advance. In the guide curve option it was possible to specify the offset value for each point of the determined guide curve, in the direction from regarded point to the probing system's central point. The negative value of this parameter moves all points of the curve closer to the central point of the probing system, and the positive one moves them away. Three cases were tested during experiment: Guide curve with offset set to $0 \mathrm{~mm},-5 \mathrm{~mm}$, and $-10 \mathrm{~mm}$ (denoted as GC 0 , GC -5 , and GC -10 , respectively). Additionally, the fixed tilt and advance method was tested for different values of tilt and advance: With a fixed tilt value of $30^{\circ}$ and three advance values, $10^{\circ}, 20^{\circ}$, and $30^{\circ}$ (denoted as FTAA $30^{\circ} / 10^{\circ}$, 
FTAA $30^{\circ} / 20^{\circ}$, and FTAA $30^{\circ} / 30^{\circ}$, respectively); and with a fixed advance value of $30^{\circ}$ and three changing tilt values, $10^{\circ}, 20^{\circ}$, and $30^{\circ}$ (denoted as FTAA $10^{\circ} / 30^{\circ}$, FTAA $20^{\circ} / 30^{\circ}$, and FTAA $30^{\circ} / 30^{\circ}$, respectively). Measurements of the diameter and circularity deviation were repeated 10 times for all described methods and cases. Results of this part of the experiment are presented in Figure 6, which shows average deviations from nominal values (taken from the calibration certificate of the standard ring), and Figure 7, which gives information about the standard deviation for each measurement task.

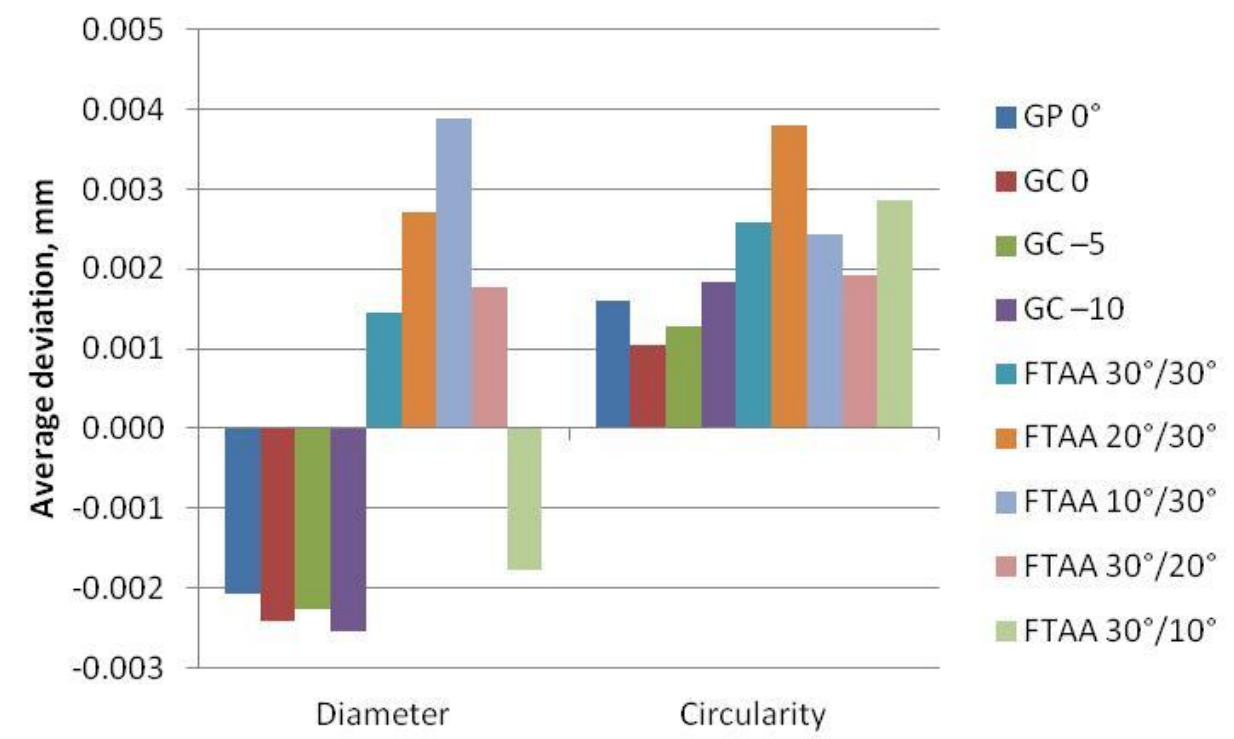

Figure 6. The average deviations for the two chosen tasks, measured on the ring standard using nine different path control algorithms.

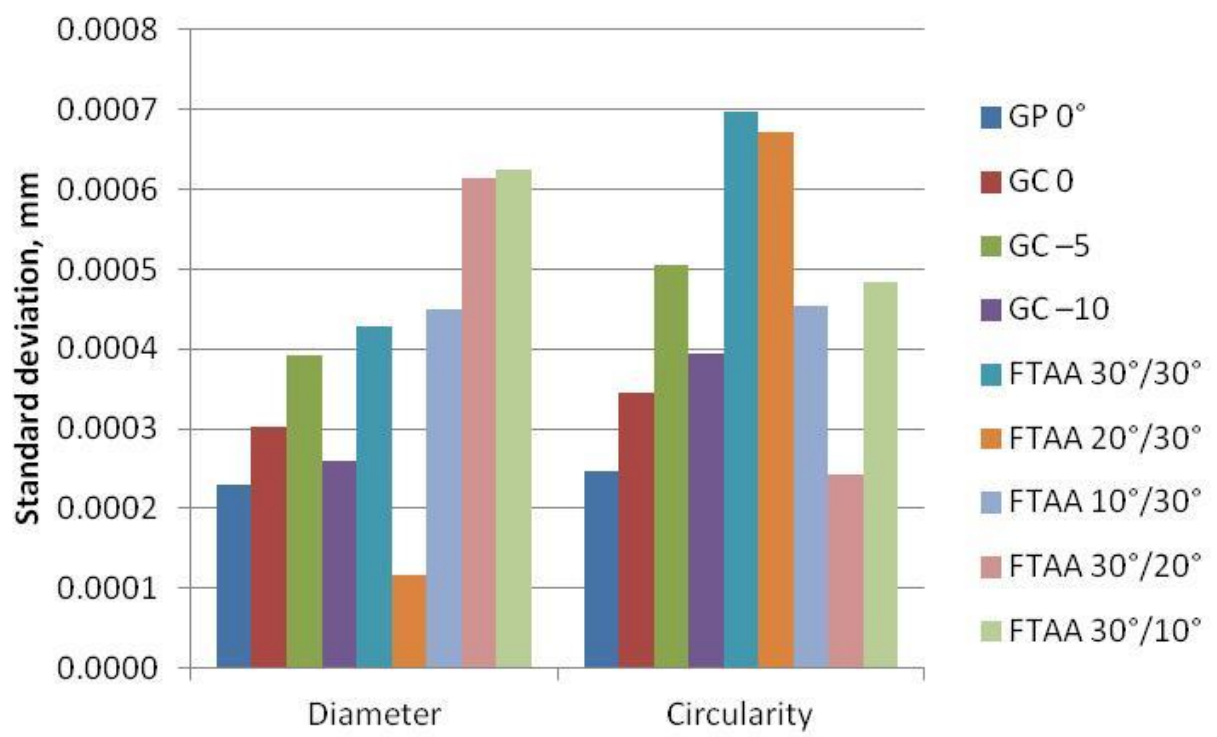

Figure 7. The standard deviations for the two chosen tasks, measured on the ring standard using nine different path control algorithms.

In the case of the standard ring measurements, the range of deviation produced by the different path control algorithms used for the determination of the diameter was equal to $6.3 \mu \mathrm{m}$, whereas for the measurement of circularity the deviation was $2.8 \mu \mathrm{m}$. What should be noticed, is that a slight change in parameter values for tilt and advance may cause significant changes in the results of both the diameter and circularity deviation measurements. 
After completing the first part of the experiments, which involved gauges measurements, the obtained results (presented in Figures 4-7) were analyzed in order to find the inspection path planning methods that gave the best results for the measurement tasks included in the experiments. The main criterion for the selection of the most suitable method was the minimization of average deviation from nominal value. Additionally, the standard deviation was also taken into account during selection, as a secondary criterion. The result of this analysis is presented in Table 2.

Table 2. The best inspection path planning method to be used for basic measuring tasks.

\begin{tabular}{cc}
\hline Measuring Task & Best Inspection Path Planning Method \\
\hline Flatness & Guide point (stylus orientation $45^{\circ}$ ) \\
Distance between planes & Guide rail \\
Parallelism & Guide point (stylus orientation $45^{\circ}$ ) \\
Angle between planes & Guide rail \\
Circularity & Guided curve (with offset set to $0 \mathrm{~mm}$ ) \\
Circle diameter & Fixed tilt and advance (tilt $30^{\circ}$; advance $30^{\circ}$ ) \\
\hline
\end{tabular}

The second part of the experiments involved measurements of chosen dimensions and relations of the multi-feature check (MFC) gauge, and determination of the relationship of the results produced by the two methods used for inspection path control: the default option (suggested by the Modus software) and the option selected on the basis of results obtained in the first part of experiments. The multi-feature check standard allows for measurements of different geometrical features and the evaluation of the diversity of geometrical dimensions and tolerances, such as distances and angles between elements, perpendicularity, parallelism, flatness, cylindricity deviations, etc. The main element forming the standard's shape was a cylinder with a length of $200 \mathrm{~mm}$ (between front planes) and an outer diameter of $100 \mathrm{~mm}$. The local coordinate system of the measured object was defined with the measurement of the external cylinder (the axis of the cylinder determines z-axis of the coordinate system), with the measurement of the plane cut into the side of the cylinder (of which the normal vector defines the $x$-axis of the coordinate system), and with the measurement of the front plane of the cylinder, which was measured in order to define the origin of the coordinate system. Next the results were compared with the actual values of the controlled dimensions taken from the MFC's calibration certificate. Calibration of the multi-feature check was performed on the Leitz PMM machine, which was located at LCM. The machine maximum permissible error Equation (1) was:

$$
E_{L, M P E}=0.8+2.5 \times L / 1000 \mu \mathrm{m}
$$

where $L$ is the measured length given in $\mathrm{mm}$.

It was located in an air-conditioned room with a thermal stability of $\pm 0.05{ }^{\circ} \mathrm{C}$. The LCM is laboratory accredited, by the Polish Centre for Accreditation, for calibration of artifacts using the coordinate measuring technique.

The following dimensions were checked (the default method for all measured features is guide point and the best method is given in brackets after each task described): The distance between the front planes of the gauge (GR); the angle between the front and side planes (GR); the parallelism deviation between the front planes of the gauge (GP $\left.45^{\circ}\right)$; the flatness of the front plane (GP $\left.45^{\circ}\right)$; and the circularity (GC 0 ) and diameter of the circle (FTAA $30^{\circ} / 30^{\circ}$ ).

Figures 8 and 9 show the results obtained during this part of the experiment. 


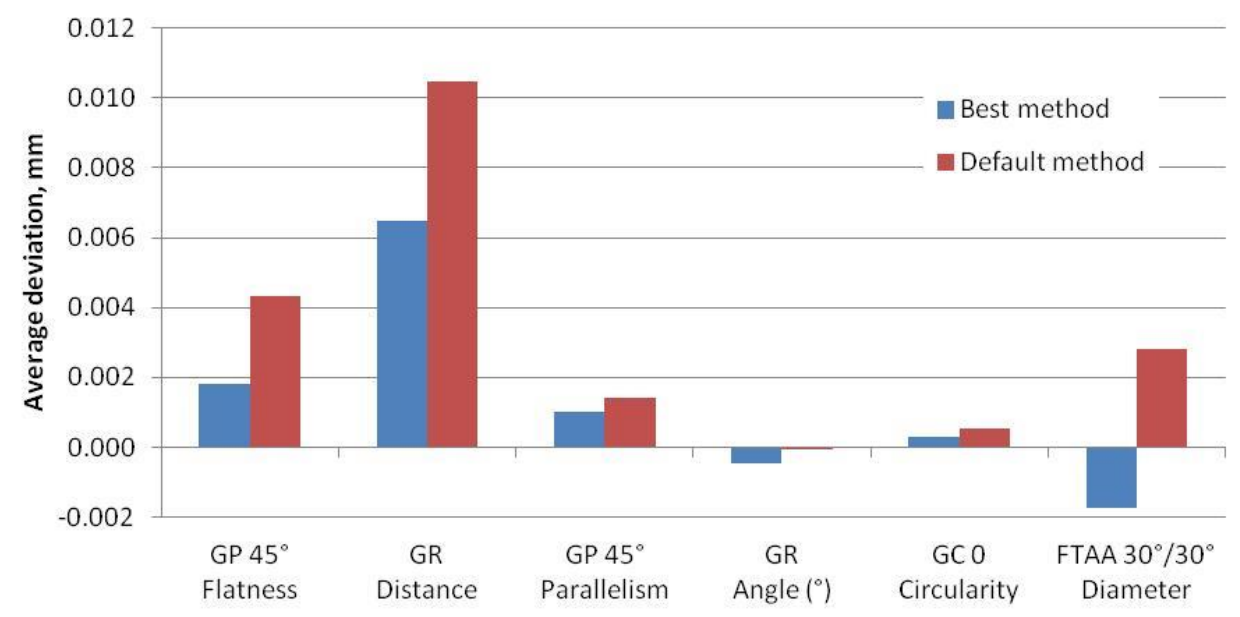

Figure 8. The average deviations for the measurements of the multi-feature check using the default and the best path control algorithms.

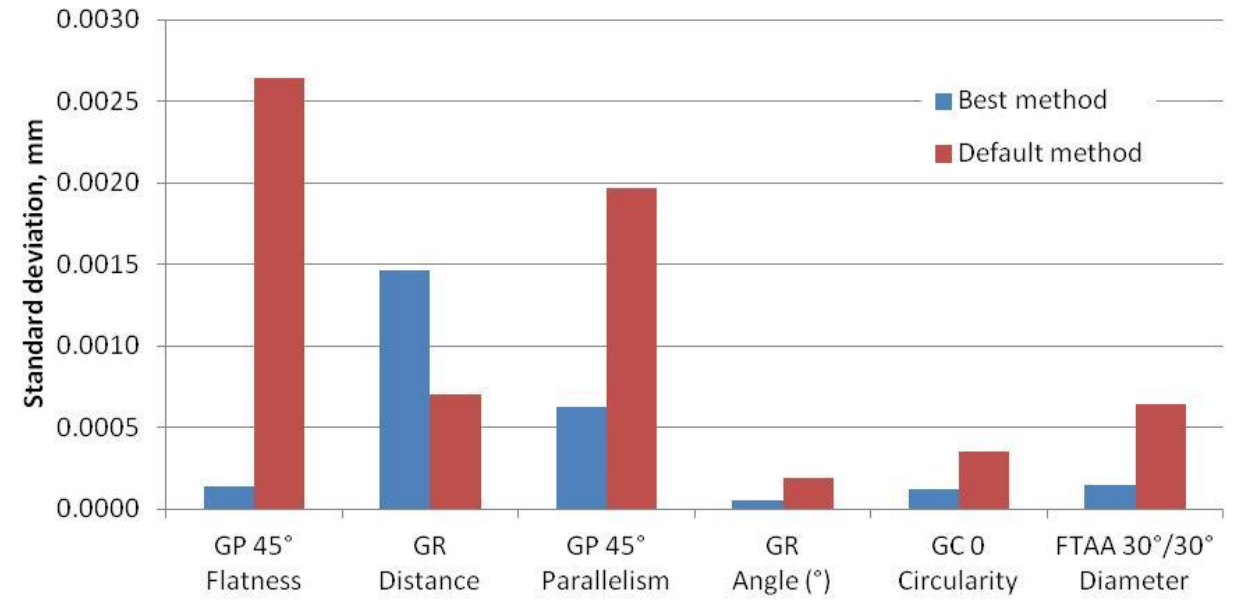

Figure 9. The standard deviations for the measurements of the multi-feature check using the default and the best path control algorithms.

\section{Discussion}

The results of the investigations presented in this paper confirmed that it is possible to reduce the measurement errors (an error is taken as the difference between the measurement result and the reference value, represented as a result of the measured workpiece calibration) using a relevant path control algorithm for the probe head used on a five-axis coordinate measuring system. In the case of some measuring tasks (e.g., distance between planes and flatness deviation), the reduction of error is obvious and reaches up to $4 \mu \mathrm{m}$; however, in the case of other (e.g., parallelism deviation and circularity deviation) it is not that significant, but the tendency to reduce the measurement error is still observed if the proper path control algorithm is used. When analyzing the values of standard deviations associated to each measuring task, it may also be observed that their values are smaller for measurements performed using the optimal path control algorithm (except one case, which is the measurement of distance between planes).

It is hard to give one exact reason why there are such big differences in results obtained using different path control algorithms. As shown, the results of the previous research undertaken by the authors in [15] showed that the accuracy of the measurements performed using the probe heads used on five-axis CMMs is strongly dependent on the orientation (the A and B angles) of the probe head during point measurement. In the majority of the presented path control algorithms, with the change of the algorithm's control parameters (e.g., tilt, advance, and offset), the probe orientation during measurement is also changing, so this may be the main cause of the differences in results. The first 
analysis confirmed this assumption; however, more detailed studies are now underway in order to unequivocally prove it. What is most important is that, as was mentioned above, it is possible to choose the best path control algorithm for each measuring task and thus reduce the measurement error.

The next observation that can be made is that it is not as important to select the relevant path control algorithm for the type of measuring feature, but rather for the type of measuring task. Therefore, for some measuring tasks, which consist of measurements of the same features (e.g., distance between two planes and parallelism deviation between them-both of these tasks consist of measurement of the same planes) but also aim to determine the different relations between them, it may occur that the measurement errors for each task are smaller if both features are measured using different path control algorithms.

The considerations presented in this paper provides guidance on the selection of the best possible path control algorithm for chosen measuring tasks (Table 2). Use of them may contribute to the general reduction of measurement error for measurements performed on five-axis systems, and the improvement of awareness of its users regarding how to run the measurements on them. This, in turn, could cause a decrease in the number of faulty classified parts during the assessment of the conformity with their geometrical specifications.

The future works on this subject will involve the preparation of complete guidelines on the selection of the best path control algorithm for chosen metrological software (preferably Modus software, which is dedicated to be used on five-axis coordinate measuring systems). It will give guidance on the selection of proper algorithms during the measurement of all measuring tasks that are able to be evaluated using this software, including all possible combinations used for solving considered tasks. In future, the proposed guidelines could also be used by the companies responsible for the development of metrological software, in order to set the default measurements of the path control algorithms that will give the lowest measurement errors.

Author Contributions: All authors conceived and designed the experiments; A.G, P.G., and M.G. performed the experiments; M.G., A.G., and P.G. analyzed the results; and P.G., A.G., and M.G. wrote the paper.

Funding: This research was funded by the National Science Centre, Poland, as part of a project grant no.: 2015/17/D/ST8/01280.

Conflicts of Interest: The authors declare no conflicts of interest.

\section{References}

1. Majchrowski, R.; Grzelka, M.; Wieczorowski, M.; Sadowski, Ł.; Gapiński, B. Large area concrete surface topography measurements using optical 3D scanner. Metrol. Meas. Sys. 2015, 22, 565-576. [CrossRef]

2. Kohut, P.; Kurc, K.; Szybicki, D.; Cioch, W.; Brudzik, R. Vision-based motion analysis and deflection.mesurement of a robot's crawler unit. J. Vibroeng. 2015, 17, 4112-4122.

3. Gapinski, B.; Wieczorowski, M.; Grzelka, M.; Alonso, P.A.; Tomé, A.B. The application of micro computed tomography to assess quality of parts manufactured by means of rapid prototyping. Polimery 2017, 62, 53-59. [CrossRef]

4. Kohut, P.; Holak, K.; Martowicz, A. An uncertainty propagation in developed vision based measurement system aided by numerical and experimental tests. J. Theo. Appl. Mech. 2012, 50, 1049-1061.

5. Delčev, S.; Gučević, J.; Ogrizović, V. Necessity of involvement of calibration laboratories in proficiency testing schemes. In Proceedings of the 11th IMEKO TC14 International Symposium on Measurement and Quality Control, Krakow /Kielce, Poland, 11-13 September 2013.

6. Zhao, F.; Xu, X.; Xie, S.Q. Computer-aided inspection planning-the state of the art. Comp. Indust. 2009, 60, 453-466. [CrossRef]

7. Lin, Y.-J.; Murugappan, P. New algorithm for CAD-directed CMM dimensional inspection. Int. J. Adv. Manuf. Technol. 2000, 16, 107-112. [CrossRef]

8. Hwang, C.Y.; Tsai, C.Y.; Chang, C.A. Efficient inspection planning for coordinate measuring machines. Int. J. Adv. Manuf. Technol. 2004, 23, 732-742. [CrossRef]

9. ISO 10360-5:2010; International Organization for Standardization (ISO): Geneva, Switzerland, 2010. 
10. Woźniak, A.; Jankowski, M. New method of testing of the repeatability of CMM articulating heads. Int. J. Adv. Manuf. Technol. 2011, 56, 677-682. [CrossRef]

11. Miguel, P.C.; King, T.; Abackerli, A. A review on methods for probe performance verification. Measurement 1998, 23, 15-23. [CrossRef]

12. Ratajczyk, E.; Woźniak, A. Coordinate Measuring Systems (In Polish: Wspótrzędnościowe Systemy Pomiarowe); Warsaw University of Technology Publishing House: Warsaw, Poland, 2016.

13. Woźniak, A.; Dobosz, M. Metrological feasibilities of CMM touch trigger probes. Part I: 3D theoretical model of probe pretravel. Measurement 2003, 34, 273-286. [CrossRef]

14. Gaska, P.; Gąska, A.; Gruza, M. Challenges for modeling of five-axis coordinate measuring systems. Appl. Sci. 2017, 7, 803. [CrossRef]

15. Gaska, A.; Gaska, P.; Gruza, M. Simulation model for correction and modeling of probe head errors in five-axis coordinate systems. Appl. Sci. 2016, 5, 144. [CrossRef]

16. MODUS ${ }^{\mathrm{TM}}$ v1.4 SP1User Guide; Renishaw: Wotton-under-Edge, UK, 2011.

(C) 2018 by the authors. Licensee MDPI, Basel, Switzerland. This article is an open access article distributed under the terms and conditions of the Creative Commons Attribution (CC BY) license (http:// creativecommons.org/licenses/by/4.0/). 